Transcontinentales

continentales

Sociétés, idéologies, système mondial

$7 \mid 2009$

Varia

\title{
Présence française au Pérou
}

Pascal RIVIALE, Una historia de la presencia francesa en el Perú, 2008

Olga L. Gonzalez

\section{OpenEdition}

1 Journals

Édition électronique

URL : http://journals.openedition.org/transcontinentales/230

DOI : 10.4000/transcontinentales.230

ISBN : 978-2-7351-1559-4

ISSN : 1775-397X

Éditeur

Editions de la maison des sciences de l'homme

\section{Édition imprimée}

Date de publication : 30 juin 2009

Pagination : 147-149

ISBN : 978-2-200-92512-3

ISSN : $1950-1684$

\section{Référence électronique}

Olga L. Gonzalez, «Présence française au Pérou », Transcontinentales [En ligne], 7 | 2009, mis en ligne le 24 mars 2011, consulté le 25 septembre 2020. URL : http://journals.openedition.org/

transcontinentales/230 ; DOI : https://doi.org/10.4000/transcontinentales.230 
Présence française au Pérou

\section{Pascal Riviale, \\ Una historia de la presencia francesa en el Perú : del siglo de las Luces a los años locos, Lima, Institut français d'études andines (Travaux de l'IFEA; 262) - Instituto de estudios peruanos - Fondo Editorial del Congreso del Perú - Embajada de Francia, 2008, 244 p.}

Contrairement à d'autres grands pays européens, comme l'Italie ou l'Espagne, la France n'a pas été un grand pays d'émigration internationale aux $\mathrm{XIX}^{e}$ et $\mathrm{XX}^{e}$ siècles. La grande vague d'émigration des Italiens et des Espagnols vers les pays du cône sud de l'Amérique latine est assez connue. Plusieurs travaux ont illustré de quelle manière les Européens, arrivés par millions dans un laps de temps très court, contribuèrent à forger les cultures nationales, en façonnant les comportements sociaux, en y imprégnant leurs modes de faire de la politique.

La faiblesse française en termes de peuplement de ces territoires n'implique pas, cependant, l'inexistence complète de projets d'installation ou l'absence d'influences commerciales, politiques et intellectuelles, comme l'attestent les travaux de Bernard Lavallé sur l'émigration aquitaine en Amérique latine, ou ceux de Denis Rolland pour le $\mathrm{xx}^{\mathrm{e}}$ siècle. En dépit du relatif désintérêt de la France vis-à-vis de l'Amérique latine au $\mathrm{XIX}^{\mathrm{e}}$ siècle, elle est restée - et demeure une référence.

Plutôt que de parler d'immigration, Pascal Riviale a choisi d'évoquer la présence des Français au Pérou. Il s'agit, d'abord, d'identifier les ressortissants français entre le Siècle des lumières et les Années folles, puis de retracer leur histoire et leur influence. Tout au long de la période, cette présence est certes manifeste, mais surtout variable. Des témoignages existent dès l'époque coloniale. Comme dans d'autres pays latinoaméricains, elle est attestée durant les luttes pour l'Indépendance auxquelles prirent part des cadres militaires français, anciens officiers de Napoléon. C'est cependant, au milieu du XIX ${ }^{e}$ siècle, lors de l'essor de l'exploitation des ressources naturelles, qu'elle atteint son apogée, et précisément au moment de la fièvre du guano. Des compagnies françaises participent très activement à ce commerce, et certains des noms mythiques de la présence française au Pérou (le chimiste Alexandre Cochet, les commerçants Auguste Dreyfus et Achille Allier) restent associés à ce moment.

Le livre comporte sept chapitres. Le premier expose les principaux événements d'ordre social, économique et politique qui ont scandé la venue des Français au Pérou. Il donne également les ordres de grandeur de cette présence (très faible pendant l'époque coloniale, à son maximum au milieu du $\mathrm{XIX}^{\mathrm{e}}$ siècle, en déclin inexorable après la guerre du Pacifique, et encore plus après la première guerre mondiale). Pour établir ce tableau, l'auteur a réalisé un minutieux travail d'enquête dans différents fonds d'archives (archives des consulats, archives civiles, recensement des étrangers, archives de la police, comptes rendus de l'Archevêché et des loges de francs-maçons, etc). Les cinq chapitres suivants s'organisent en fonction des principales activités et métiers exercés par ces Français : artisans commerçants et industriels; professions libérales et enseignants; archéologues et scientifiques; artistes; membres des congrégations religieuses. Enfin, le dernier chapitre détaille les pratiques associatives créées pour maintenir l'identité française, mais aussi pour tisser des liens avec la société péruvienne. 
Malgré sa faiblesse relative - durant cette période les Français n'ont jamais dépassé quelques milliers de personnes - l'influence de la communauté se fit sentir de manière remarquable. C'est notamment le cas dans les secteurs d'activité où les Français étaient numériquement le plus représentés, c'est-à-dire en premier lieu le commerce et certaines branches de l'artisanat. On ne s'étonnera pas de les voir apparaître dans les secteurs de la restauration et de l'hôtellerie, de les compter parmi les premiers cultivateurs de vignobles péruviens et plus largement dans la cuisine (saiton qu'un des plus célèbres cafés de Lima - le Café anglais, fondé en 1868 - était tenu par des Français?). On les rencontre également dans le secteur de la mode (la seule mention de Paris sur les devantures semblait apporter l'aura de l'élégance française), en tant que propriétaires de boutiques, ou parmi les représentants des grands magasins.

Les Français sont également présents dans les grands travaux. Des ingénieurs et des architectes français ont laissé des œuvres, des agronomes et des chimistes ont contribué à apporter des progrès techniques. Des spécialistes ont été sollicités par le gouvernement péruvien dans le domaine de l'éducation, un terrain investi également par les religieuses (les sœurs de Picpus, les sœurs de Cluny). Plusieurs de ces institutions ont posé les jalons d'un enseignement ouvert à de nouvelles méthodes et elles ont joui d'un prestige qui perdure de nos jours. Dans le domaine militaire, des traités de coopération entre les armées des deux pays se concrétisèrent au tournant du $\mathrm{xx}^{\mathrm{e}}$ siècle dans la formation des officiers péruviens.

Pascal Riviale nous apprend que les expéditions scientifiques ont débuté très tôt. Ainsi, les premiers Français à avoir touché les côtes péruviennes - les commerçants de Saint-Malo -, après s'être reconvertis dans la contrebande en raison des restrictions imposées par les autorités espagnoles, ont été amenés à créer des compagnies qui servirent, par la suite, aux expéditions scientifiques. En effet, dès la fin du $\mathrm{XVII}^{\mathrm{e}}$ siècle, et pour des raisons en partie d'ordre stratégique, le roi Louis XIV avait favorisé et soutenu des expéditions destinées à effectuer les relevés géographiques des différents points de la côte. Ces expéditions ont parfois donné lieu à des découvertes dont l'impact fut retentissant : au $\mathrm{XIX}^{e}$ siècle, les pièces envoyées par ces archéologues avant l'heure, souvent des diplomates ou des militaires affectés au Pérou, expliquent l'engagement grandissant des ministères et institutions françaises. Ainsi, la décision de fonder le musée du Trocadéro fut prise après la présentation mémorable de la mission de Charles Wiener à l'Exposition universelle de Paris en 1879, pour abriter les pièces rapportées.

Bien que l'auteur estime le bilan de la présence française largement positif, il ne manque pas de souligner les difficultés et les incompréhensions rencontrées par ces immigrants. Il arrivait qu'on les identifiât avec une "modernité» qui représentait à la fois une promesse et une menace. Ainsi, les idées proposées par un médecin français pour le traitement des malades lui valurent l'hostilité de ses collègues. Les projets commerciaux et industriels se sont souvent soldés par des échecs (l'auteur rappelle les mésaventures de l'homme d'affaires Félix Dibois, importateur d'armes, entrepreneur dans des projets de développement touristique et ferroviaire, et qui finit ses jours à Lima, ruiné). Le manque de soutien en France ou au Pérou, les difficultés dans 
le suivi des travaux (comme dans le cas de certaines missions scientifiques pourtant appuyées par les ministères français), les aléas (ainsi la mort du partenaire de Gustave Eiffel au Pérou mit brutalement fin à ses projets) ont produit leur lot de frustrations. Dans ce sens, les enquêtes menées dans d'autres pays - comme l'étude monographique de Pierre Raymond sur l'établissement et l'échec d'un très ambitieux projet industriel franco-belge-colombien - devraient être un filon tout aussi révélateur pour saisir la portée de ces difficultés.

Pascal Riviale s'arrête tout particulièrement sur l'absence d'une immigration de type agricole (hormis le cas exceptionnel de Chanchamayo). Comme le montrent les travaux de Dora Estela Celton sur l'échec de la colonisation en Argentine, les mesures proclamées par les gouvernements de la plupart des pays d'Amérique latine au $\mathrm{XIX}^{e}$ siècle afin de favoriser l'implantation de colons européens dans des territoires semi-déserts n'ont finalement eu que des répercussions limitées. La politique volontariste en matière d'immigration se heurtait à la réalité, toujours plus complexe que les desiderata de la sphère politique.

Enfin, le livre dégage des phénomènes d'ordre structurel bien connus des spécialistes des migrations. Le cas du canton de Saint-Gaudins, dans les Pyrénées, cité comme lieu d'origine de nombreux ressortissants français tout au long du $\mathrm{XIX}^{\mathrm{e}}$ siècle, montre que de tels phénomènes relèvent de stratégies en réseaux facilitant les projets de migration. Ces dynamiques sont observées aujourd'hui dans différentes régions du monde.

Plus largement, les motivations qui poussèrent des milliers d'individus à émigrer très loin de chez eux, à la recherche d'un Pérou imaginaire, ne doiventelles pas être rapprochées de celles qui sont avancées aujourd'hui par d'autres migrants? À commencer, bien sûr, par de nombreux Andins, aujourd'hui installés en Espagne, en France ou dans d'autres pays, qui poursuivent eux aussi leur quête d'un eldorado.

En somme, il se dégage de ce panorama une image d'ensemble riche de l'immigration française au Pérou, même si celle-ci est restée faible numériquement. Ce type de travail peut donner lieu à des développements ultérieurs. Il pourrait, par exemple, permettre de resituer la présence et l'influence françaises dans le contexte plus large de l'immigration européenne en Amérique latine, ou bien être complété par des recherches portant sur la portée des idées françaises (dans une démarche analogue à celle de Frédéric Martinez pour la Colombie). Enfin, l'intérêt de ce travail réside aussi dans le fait qu'il met particulièrement en lumière des processus et des enjeux du temps présent.

Olga L. Gonzalez Unité de recherches Migrations et sociétés (URMIS), université Paris-VIII Groupe Actualités Colombie (GAC), Fondation Maison des sciences de l'homme 